19

\title{
Анализ влияния нелокальности на характеристики ближнего поля слоистой частицы на подложке
}

\author{
(C) Ю.А. Еремин \\ Московский государственный университет им. М.В. Ломоносова, \\ 119991 Москва, Россия \\ e-mail: eremin@cs.msu.ru
}

Поступила в редакцию 28.04.2020 г.

В окончательной редакции 28.04.2020 г.

Принята к публикации 29.05.2020 г.

Рассмотрена задача дифракции поля электромагнитной плоской волны на слоистой наночастице с металлическим плазмонным слоем, расположенной на поверхности прозрачной подложки. На основе метода дискретных источников исследовано влияния пространственной нелокальности в металле на интенсивность ближнего поля и сечение поглощения. Рассмотрены случаи возбуждения частицы как распространяющейся, так и неизлучающей волной. Показано, что подложка оказывает более существенное влияние на оптические характеристики ближнего поля, чем на интенсивность в дальней зоне. Установлено, что учет эффекта нелокальности в металле приводит к существенному снижению амплитуды плазмонного резонанса при небольшом сдвиге в коротковолновую область.

Ключевые слова: метод дискретных источников, плазмонный резонанс, слоистая наночастица.

DOI: $10.21883 /$ OS.2020.09.49881.141-20

\section{Введение}

Квантовая наноплазмоника привлекает все больший интерес у исследователей во многих странах. Как известно, поверхностные плазмоны позволяют концентрировать электромагнитные поля в субнанометровых объемах, значительно превышая классический предел оптической дифракции. Эта область плазмоники интенсивно развивается благодаря совершенствованию технологий синтеза наноразмерных структур, которые ведут к миниатюризации элементов фотонных схем. Последнее обстоятельство создает условия для их использования в многочисленных практических приложениях [1].

За последние десятилетия использование слоистых наночастиц приобрело огромную популярность благодаря возможности манипулирования материалами и размерами как ядра, так оболочки для управления их плазмонными свойствами. Это обстоятельство выгодно отличает их от однородных частиц. Благодаря впечатляющему прогрессу в материаловедении слоистые наночастицы теперь могут быть синтезированы с улучшенными физико-химическими свойствами, четко определенными размерами, формой и составом [2]. Используя их способность в сверхвысоком усилении поля и концентрации субволнового поля, стало возможным широкое разнообразие применений во многих областях промышленного, клинического, биологического, экологического и пищевого анализа [3,4]. Особый интерес в наноплазмонике вызывает проблема разработки и реализации плазмонного нанолазера. Плазмонные нанолазеры, основанные на использовании слоистых резонаторов, имеют преимущества наноразмера, низкого энергетического порога и супермалого времени отклика [5].
В целом ряде приложений, упомянутых выше, возникает необходимость в исследовании оптических свойств наночастиц, расположенных на диэлектрической подложке. В этом случае весьма важно строго учитывать взаимодействие между частицей и поверхностью подложки. Эти обстоятельства возникают как при попытках обнаружения и определения структуры привнесенных частиц, так и решении проблем синтеза слоистых структур для получения требуемых спектральных свойств [6,7]. При сокращении размера металлических элементов до десятка нанометров электрон-электронные взаимодействия в наноструктурах должны учитываться на другом уровне. Дело в том, что когда характерный размер металлических наночастиц становится сравнимым с длиной волны Ферми электронов в этом металле ( $5 \mathrm{~nm}$ для золота и серебра), возникает так называемая пространственная нелокальность металла. В этом случае классической системы уравнений Максвелла оказывается недостаточно для точного описания электромагнитных свойств плазмонного металла [1].

Существуют различные подходы к описанию возникающего эффекта нелокальности (ЭН), начиная с полной квантово-механической модели, в рамках которой моделируется поведение каждого электрона наноструктуры на основе уравнения Шредингера. Она хорошо подходит для объяснения экспериментальных результатов, но применима лишь к плазмонным частицам диаметром всего в несколько нанометров [8]. В настоящее время при исследовании наночастиц используются подходы, которые учитывают возникающие квантовые эффекты, но позволяют описать их в рамках электромагнитной теории Максвелла. Одним из таких полуклассических подходов, учитывающих ЭН в металлах, является гид- 
родинамическая модель Друде $[9,10]$. Она позволяет учитывать возникающие в металле продольные поля, но требует корректировки квантовых параметров металла при рассмотрении однородных частиц, форма которых отличается от сферической [11]. Следующая модель, свободная от этого ограничения, получила название обобщенного нелокального оптического отклика (ОНО) [12]. В рамках ОНО учитывается диффузия электронов в металле в рамках гидродинамической теории. Это обстоятельство дает возможность исследовать как однородные несферические частицы, так и их кластеры. Модель ОНО оказалась подходящим инструментом также для исследования оптических свойств сферических слоистых частиц в свободном пространстве [13]. Теория ОНО позволяет учитывать как возникающие внутри металла продольные поля, так и дополнительные граничные условия на границах раздела между металлом и диэлектриком [14].

Мы используем модель ОНО в рамках метода дискретных источников (МДИ) [15]. Метод дискретных источников - строгий численно-аналитический поверхностно ориентированный метод. Он основан на представлении полей в виде конечной линейной комбинации распределенных мультиполей низшего порядка [16], удовлетворяющих полуклассическим уравнениям Максвелла, включая продольные поля, внутри металлической оболочки. Для представления рассеянных полей вне частицы используется тензор Грина полупространства [17]. Таким образом, представления полей во всех областях удовлетворяют уравнениям Максвелла, условиям бесконечности и условиям сопряжения на бесконечной поверхности призмы. Соответствующие амплитуды дискретных источников определяются из условий сопряжения, поставленных на поверхностях слоистой частицы, включая дополнительные граничные условия. Исключительная особенность МДИ состоит в том, что он позволяет оценить реальную погрешность полученного решения посредством вычисления невязки полей на поверхностях слоев. Данное обстоятельство дает возможность вычислять ближние поля с гарантированной точностью, что особенно существенно при анализе усиления интенсивности вблизи частицы или сечения поглощения. Все эти моменты позволили использовать МДИ для анализа плазмонных наноструктур с учетом эффекта нелокальности в рамках модели ОНО $[18,19]$.

\section{Постановка граничной задачи дифракции, модель ОНО}

Пусть все пространство $\Re^{3}$ разделено на два полупространства: вмещающая среда $-D_{0}:(z>0)$ и диэлектрическая подложка $-D_{1}:(z<0)$. Обозначим $\Sigma:(z=0)$ плоскую границу раздела. Пусть сферическая слоистая частица целиком располагается в верхнем полупространстве $D_{0}$. Внутреннее диэлектрическое ядро частицы будем обозначать как $D_{i}$, а область металлической оболочки - как $D_{s}$. Соответствующие сферические поверхности обозначим как $\partial D_{i, s}$. Все среды предполагаются немагнитными, а их диэлектрические проницаемости обозначим $\varepsilon_{v}, v=0,1, i, s$.

Суть ЭН заключается в том, что связь между смещением $\mathbf{D}(M)$ и напряженностью электрического поля $\mathbf{E}(M)$ становится нелокальной, т.е. соотношение $\mathbf{D}(M)=\varepsilon(M) \mathbf{E}(M)$ заменяется на

$$
\mathbf{D}(M)=\int \varepsilon\left(M-M^{\prime}\right) \mathbf{E}\left(M^{\prime}\right) d M^{\prime},
$$

которое переходит в локальное в случае $\varepsilon\left(M-M^{\prime}\right)=\delta\left(M-M^{\prime}\right) \quad[14]$. Одним из проявлений ЭН является появление внутри металла продольных электромагнитных полей. В этом случае внутреннее электрическое поле Е перестает быть чисто поперечным $\left(\operatorname{div} \mathbf{E}^{T}=0\right)$ за счет формирования объемного заряда, и для адекватного описания происходящих процессов возникает необходимость привлечение дополнительно продольных полей $\left(\operatorname{rot} \mathbf{E}^{L}=0\right) \quad[20]$. Для учета нелокальности используется гидродинамическая теория Друде и ее обобщение - модель ОНО [12]. В рамках теории ОНО проводится обобщение закона Ома для тока проводимости внутри металла, т. е. осуществляется переход следующего вида:

$$
\mathbf{J}=\sigma \mathbf{E} \Rightarrow \xi^{2} \operatorname{grad}(\operatorname{div} \mathbf{J})+\mathbf{J}=\sigma \mathbf{E},
$$

где $\sigma$ - проводимость металла, $\xi$ - параметр нелокальности [14]. В результате изменяется соответствующее уравнение системы Максвелла для магнитного поля. В силу изложенных обстоятельств внутри плазмонного металла происходит расщепление электрического поля на поперечное и продольное: $\mathbf{E}_{s}=\mathbf{E}_{s}^{T}+\mathbf{E}_{s}^{L}, \operatorname{div} \mathbf{E}_{s}^{T}=0$, $\operatorname{rot} \mathbf{E}_{s}^{L}=0$. Можно показать [21], что эти поля внутри оболочки $D_{s}$ удовлетворяют уравнениям Гельмгольца:

$$
\begin{gathered}
\Delta \mathbf{E}^{T}(M)+k_{T}^{2} \mathbf{E}^{T}(M)=0, \\
\Delta \mathbf{E}^{L}(M)+k_{L}^{2} \mathbf{E}^{L}(M)=0 .
\end{gathered}
$$

Здесь $k_{T}^{2}=k^{2} \varepsilon_{s}, k_{L}^{2}=\varepsilon_{s} / \xi-$ поперечное и продольное волновые числа.

Перейдем к формулировке математической постановки граничной задачи рассеяния для системы Максвелла с учетом ОНО. Обозначим $\left\{\mathbf{E}^{0}, \mathbf{H}^{0}\right\}$ поле плоской электромагнитной волны линейной поляризации, распространяющейся из верхнего полупространства в полуплоскости $\varphi=\pi$ под углом $\pi-\theta_{0}$ относительно нормали к подложке, совпадающей с осью $0 z$. Тогда постановка граничной задачи рассеяния с учетом ЭН может быть записана в следующем виде:

$$
\begin{gathered}
\operatorname{rot} \mathbf{H}_{\zeta}=j k \varepsilon_{\zeta} \mathbf{E}_{\xi} ; \operatorname{rot} \mathbf{E}_{\xi}=-j k \mathbf{H}_{\xi} \text { в области } D_{\xi}, \\
\xi=0,1, i, \\
\operatorname{rot} \mathbf{H}_{s}=j k\left(\varepsilon_{s}+\xi^{2} \operatorname{graddiv}\right) \mathbf{E}_{s}(M) ; \operatorname{rot} \mathbf{E}_{s}=-j k \mathbf{H}_{s} \\
\text { в области } D_{s},
\end{gathered}
$$




$$
\begin{gathered}
\mathbf{n}_{i} \times\left(\mathbf{E}_{i}(P)-\mathbf{E}_{s}(P)\right)=0, \\
\mathbf{n}_{i} \times\left(\mathbf{H}_{i}(P)-\mathbf{H}_{s}(P)\right)=0, P \in \partial D_{i} ; \\
\varepsilon_{i} \mathbf{n}_{i} \cdot \mathbf{E}_{i}(P)=\varepsilon_{L} \mathbf{n}_{i} \cdot \mathbf{E}_{s}(P) \\
\mathbf{e}_{z} \times\left(\mathbf{E}_{0}(Q)-\mathbf{E}_{1}(Q)\right)=0, Q \in \Sigma . \\
\mathbf{e}_{z} \times\left(\mathbf{H}_{0}(Q)-\mathbf{H}_{1}(Q)\right)=0, \\
\mathbf{n}_{l} \times\left(\mathbf{E}_{s}(P)-\mathbf{E}_{0}^{s}(P)\right)=\mathbf{n}_{s} \times \mathbf{E}_{0}^{0}(P), \\
\mathbf{n}_{l} \times\left(\mathbf{H}_{s}(P)-\mathbf{H}_{0}^{s}(P)\right)=\mathbf{n}_{s} \times \mathbf{H}_{0}^{0}(P), P \in \partial D_{s} ; \\
\varepsilon_{L} \mathbf{n}_{s} \cdot \mathbf{E}_{s}(P)=\varepsilon_{0} \mathbf{n}_{s} \cdot\left(\mathbf{E}_{0}^{0}(P)+\mathbf{E}_{0}^{s}(P)\right) \\
\lim _{r \rightarrow \infty} r \cdot\left(\mathbf{H}_{0}^{s} \times \frac{\mathbf{r}}{r}-\sqrt{\varepsilon_{0}} \mathbf{E}_{0}^{s}\right)=0, \\
r=|M| \rightarrow \infty, \xi=0,1, z \neq 0 ; \\
\max \left(\left|\mathbf{H}_{0}^{s}\right|,\left|\mathbf{E}_{0}^{s}\right|\right)=O(\rho-1 / 2), \rho=\sqrt{x^{2}+y^{2}}, \\
\rho \rightarrow \infty, z= \pm 0 .
\end{gathered}
$$

Здесь $\left\{\mathbf{E}_{\xi}, \mathbf{H}_{\xi}\right\}-$ полные поля в пространстве $D_{\xi}$, $\zeta=0,1, i, s$ соответственно, $\mathbf{n}_{i, s}$ - единичные нормали к поверхностям $\partial D_{i s}, \mathbf{e}_{z}-$ нормаль в поверхности подложки, $k=\omega / c$, характеристики среды выбраны таким образом, что $\operatorname{Im} \varepsilon_{0,1, s}=0, \operatorname{Im} \varepsilon_{s} \leq 0, \operatorname{Im} \varepsilon_{L}=0$. Предполагается, что временная зависимость выбрана в виде $\exp \{j \omega t\}$.

Конкретизируем остальные величины, входящие в постановку задачи (4). Поля $\left\{\mathbf{E}_{\xi}^{0}, \mathbf{H}_{\xi}^{0}\right\}, \xi=0,1$ представляют собой результат решения задачи отражения и преломления поля плоской волны $\left\{\mathbf{E}^{0}, \mathbf{H}^{0}\right\}$ на плоскости раздела $\Sigma$ полупространств $D_{0,1}$. Поле $\left\{\mathbf{E}_{\xi}^{s}, \mathbf{H}_{\xi}^{s}\right\}, \xi=0,1$ есть рассеянное поле в каждом из полупространств, которое определяется, как $\mathbf{E}_{\xi}^{s}=\mathbf{E}_{\xi}-\mathbf{E}_{\xi}^{0}, \mathbf{H}_{\xi}^{s}=\mathbf{H}_{\xi}-\mathbf{H}_{\xi}^{0}$, $\xi=0,1$. В силу построения поля внешнего возбуждения и граничных условий на $\Sigma$ рассеянное поле $\left\{\mathbf{E}_{\xi}^{s}, \mathbf{H}_{\xi}^{s}\right\}$, $\xi=0,1$ также должно удовлетворять условиям сопряжения для тангенциальных компонент на бесконечной границе $\Sigma$.

На поверхностях $\partial D_{i, s}$ в дополнение к классическим условиям сопряжения поставлены дополнительные условия для нормальных компонент полей, необходимые для однозначной разрешимости задачи. Эти условия физически соответствуют условиям обращения в нуль нормальной компоненты тока проводимости на границах раздела металл-диэлектрик $\mathbf{n} \cdot \mathbf{J}=0$, которые затем трансформируются в условия для нормальных компонент полей [14]. Причем последние отличаются от стандартных условий сопряжения для нормальных компонент смещения D, которые являются следствием непрерывности тангенциальных компонент полей в классической постановке. Из формулировки задачи (4) непосредственно вытекает, что продольная компонента поля, во-первых, локализована строго внутри слоя, и вовторых, не вносит вклад в магнитное поле $\mathbf{H}_{s}$, так как $\operatorname{rot}(\operatorname{grad} \Psi) \equiv 0$. Условия излучения задачи (4) сформулированы таким образом, чтобы обеспечить обращение в нуль потока энергии на бесконечности для однородной задачи (4) [22]. Мы будем полагать, что поставленная граничная задача (4) имеет единственное классическое решение. Параметры $\xi$ и $\varepsilon_{L}$, относящиеся к продольному полю $\mathbf{E}_{s}^{L}$, определяются следующим образом:

$$
\begin{aligned}
& \varepsilon_{L}=\varepsilon_{s}-\omega_{p}^{2} /\left(j \gamma \omega-\omega^{2}\right), \\
& \quad \xi^{2}=\varepsilon_{s}\left(\beta^{2}+D(\gamma+j \omega)\right) /\left(\omega^{2}-j \gamma \omega\right) .
\end{aligned}
$$

Здесь $\omega_{p}$ - плазменная частота металла, $\gamma-$ коэффициент затухания, $\beta$ - гидродинамическая скорость в плазме, связанная со скоростью Ферми $v_{F}$ соотношением $\beta^{2}=3 / 5 v_{F}^{2}, D-$ коэффициент диффузии электронов [12].

\section{Метод дискретных источников с учетом модели ОНО}

Будем строить приближенное решение задачи (4), руководствуясь схемой [17]. Ограничимся случаем $P$-поляризации, поскольку именно она реализует наибольшую амплитуду плазмонного резонанса [23]. Так как частица целиком располагается в верхнем полупространстве $D_{0}$, то суммарное поле падающей и отраженной волн $\left\{\mathbf{E}_{0}^{0}, \mathbf{H}_{0}^{0}\right\}$ приобретает вид

$$
\mathbf{E}_{0}^{0}=\mathbf{E}_{0}^{-}+\mathbf{R}_{p} \cdot \mathbf{E}_{0}^{+} ; \mathbf{H}_{0}^{0}=\mathbf{H}_{0}^{-}+\mathbf{R}_{p} \cdot \mathbf{H}_{0}^{+} .
$$

Здесь $\left\{\mathbf{E}_{0}^{-} \mathbf{H}_{0}^{-}\right\}=\mathbf{E}^{0} \mathbf{H}^{0}, \quad \mathbf{E}_{0}^{ \pm}=\mathbf{e}_{0}^{ \pm} \psi_{0}^{ \pm} ; \quad \mathbf{H}_{0}^{ \pm}=-\mathbf{e}_{y} n_{0} \psi_{0}^{ \pm}$, $n_{0}=\sqrt{\varepsilon_{0}}, R_{P}-$ коэффициент отражения Френеля [24],

$$
\begin{gathered}
\psi_{0}^{ \pm}=\exp \left\{-j k_{0}\left(x \sin \theta_{0} \pm z \cos \theta_{0}\right)\right\}, \\
\mathbf{e}_{0}^{ \pm}=\left(\mp \mathbf{e}_{x} \cos \theta_{0}+\mathbf{e}_{z} \sin \theta_{0}\right),
\end{gathered}
$$

где $\mathbf{e}_{x}, \mathbf{e}_{y}, \mathbf{e}_{z}-$ единичные векторы декартовой системы координат.

Построим приближенное решение задачи (4) для рассеянного поля в $D_{0}$ с учетом осевой симметрии и поляризации, удовлетворяя квазиклассической системе уравнений Максвелла во всех областях постоянства параметров среды, условиям излучения и условиям сопряжения для полей на $\Sigma$. В основу представления для рассеянного поля положим фурье-компоненты тензора Грина полупространства, которые могут быть записаны в виде интегральных представлений ВейляЗоммерфельда [17]:

$$
\begin{aligned}
& G_{m}^{e, h}\left(\eta, z_{n}\right)=\int_{0}^{\infty} J_{m}(\lambda \rho) v_{11}^{e, h}\left(\lambda, z, z_{n}\right) \lambda^{1+m} d \lambda, \\
& g_{m}^{(e, h)}\left(\eta, z_{n}\right)=\int_{0}^{\infty} J_{m}(\lambda, \rho) v_{31}^{e, h}\left(\lambda, z, z_{n}\right) \lambda^{1+m} d \lambda .
\end{aligned}
$$

Здесь $J_{m}($.$) - цилиндрическая функция Бесселя, точка$ $\eta=(\rho, z)$ располагается в полуплоскости $\varphi=$ const, a точки локализации мультиполей распределены вдоль оси симметрии $z_{n} \in O Z$ строго внутри $D_{i} \cup D_{s}$. Спектральные функции электрического и магнитного типов $v_{11}^{e, h}$, 
$v_{31}^{e, h}$ обеспечивают выполнение условий сопряжения на границе интерфейса $z=0$. В данном случае для них справедливы следующие выражения:

$$
\begin{aligned}
& v_{11}^{e, h}\left(\lambda, z, z_{n}\right)=\frac{\exp \left\{-\eta_{0}\left|z-z_{n}\right|\right\}}{\eta_{0}} \\
&+A_{11}^{e, h}(\lambda) \exp \left\{-\eta_{0}\left(z+z_{n}\right)\right\}, z_{n}>0, z \geq 0 \\
& v_{31}^{e, h}\left(\lambda, z, z_{n}\right)= A_{31}^{e, h}(\lambda) \exp \left\{-\eta_{0}\left(z+z_{n}\right)\right\}, \\
& z_{n}>0, z \geq 0 .
\end{aligned}
$$

Спектральные коэффициенты $A, B$ определяются из одномерной задачи с условиями сопряжения при $z=0$, откуда легко получается, что

$$
A_{11}^{e, h}(\lambda)=\frac{\chi_{0}^{e, h}-\chi_{1}^{e, h}}{\chi_{0}^{e, h}+\chi_{1}^{e, h}} \frac{1}{\eta_{0}}, A_{31}^{e, h}(\lambda)=\frac{2 \delta}{\left(\chi_{0}^{e}+\chi_{1}^{e}\right)\left(\chi_{0}^{h}+\chi_{1}^{h}\right)},
$$

где $\eta_{\xi}=\sqrt{\lambda^{2}-k_{\xi}^{2}}, \chi_{\xi}^{e}=\eta_{\xi}, \chi_{\xi}^{h}=\eta_{\xi} / \varepsilon_{\xi}, \delta=1 / \varepsilon_{0}-1 / \varepsilon_{1}$, $\xi=0,1$.

Для построения приближенного решения для рассеянного поля в пространстве $D_{0}$ используются векторные потенциалы, которые в цилиндрической системе координат могут быть записаны как

$$
\begin{gathered}
\mathbf{A}_{m n}^{(e) 0}=\left\{G_{m}^{e}\left(\eta, z_{n}^{e}\right) \cos (m+1) \varphi ;\right. \\
\left.-G_{m}^{e}\left(\eta, z_{n}^{e}\right) \sin (m+1) \varphi ;-g_{m}^{e}\left(\eta, z_{n}^{e}\right) \cos (m+1) \varphi\right\}, \\
\mathbf{A}_{m n}^{(h) 0}=\left\{G_{m}^{h}\left(\eta, z_{n}^{e}\right) \sin (m+1) \varphi ;\right. \\
\left.G_{m}^{h}\left(\eta, z_{n}^{e}\right) \cos (m+1) \varphi ;-g_{m+1}^{h}\left(\eta, z_{n}^{e}\right) \sin (m+1) \varphi\right\}, \\
\mathbf{A}_{0 n}^{(e) 0}=\left\{0 ; 0 ; G_{0}^{h}\left(\eta, z_{n}^{e}\right)\right\} .
\end{gathered}
$$

Для построения полей внутри областей $D_{i, s}$ будут использоваться следующие потенциалы:

$$
\begin{aligned}
& \mathbf{A}_{m n}^{(e) v}=\left\{Y_{m}^{v}\left(\eta, z_{n}^{v}\right) \cos (m+1) \varphi\right. \\
& \left.-Y_{m}^{v}\left(\eta, z_{n}^{v}\right) \sin (m+1) \varphi ; 0\right\}, v=i, s \pm ;
\end{aligned}
$$

$$
\begin{aligned}
& \mathbf{A}_{m n}^{(h) v}=\left\{Y_{m}^{v}\left(\eta, z_{n}^{v}\right) \sin (m+1) \varphi\right. \\
& \left.-Y_{m}^{v}\left(\eta, z_{n}^{v}\right) \cos (m+1) \varphi ; 0\right\}, \mathbf{A}_{n}^{(e) v}=\left\{0 ; 0 ; Y_{0}^{v}\left(\eta, z_{n}^{v}\right)\right\}
\end{aligned}
$$

Здесь $Y_{m}^{i}\left(\eta, z_{n}^{i}\right)=j_{m}\left(k_{i} r_{\eta z_{n}^{i}}\right)\left(\rho / r_{\eta z_{n}^{i}}\right)^{m}, j_{m}()-$. сферическая функция Бесселя, $Y_{m}^{s \pm}\left(\eta, z_{n}^{s}\right)=h_{m}^{(2,1)}\left(k_{s} r_{\eta z_{n}^{s}}\right) \times$ $\times\left(\rho / r_{\eta z_{n}^{s}}\right)^{m}, h_{m}^{(2,1)}($.$) - сферические функция Ханкеля,$ соответствующие ,уходящим“ и „приходящим“ волнам, $r_{\eta z_{n}}^{2}=\rho^{2}+\left(z-z_{n}\right)^{2}, \quad \eta=(\rho, z), \quad k_{i s}=k \sqrt{\varepsilon_{i, s}}, \quad z_{n}^{i, s}-$ координаты дискретных источников (ДИ). Следует отметить, что функции, положенные в основу векторных потенциалов (7), (8), удовлетворяют уравнению Гельмгольца (2).

Для случая $P$-поляризации продольное поле строится на основе следующих скалярных потенциалов [18]:

$$
\begin{gathered}
\Psi_{m n}^{s \pm}(M)=h_{m+1}^{(2,1)}\left(k_{L} R_{\eta z_{n}^{s}}\right) P_{m+1}^{m+1}\left(\cos \theta_{z_{n}^{s}}\right) \cos (m+1) \varphi \\
\Psi_{n}^{s \pm}(M)=h_{0}^{(2,1)}\left(k_{L} R_{\xi z_{n}^{s}}\right),
\end{gathered}
$$

которые удовлетворяют уравнению Гельмгольца (3). Тогда приближенное решение для полного поля внутри частицы и рассеянного поля в пространстве $D_{0}$, соответствующее $P$-поляризации, принимает следующий вид:

$$
\begin{aligned}
\mathbf{E}_{v}^{T N} & =\sum_{m=0}^{M} \sum_{n=1}^{N_{v}^{m}}\left\{p_{m n}^{v} \frac{j}{k \varepsilon_{v}} \operatorname{rotrot} \mathbf{A}_{m n}^{(e) v}\right. \\
& \left.+q_{m n}^{v} \frac{1}{\varepsilon_{v}} \operatorname{rot} \mathbf{A}_{m n}^{(h) v}\right\}+\sum_{n=1}^{N_{v}^{0}} r_{n}^{v} \frac{j}{k \varepsilon_{v}} \operatorname{rot} \operatorname{rot} \mathbf{A}_{n}^{(e) v} ; \\
\mathbf{E}_{s \pm}^{L N}= & \sum_{m=0}^{M} \sum_{n=1}^{N_{s}^{m}} \bar{p}_{m m}^{s \pm} \operatorname{grad} \Psi_{m n}^{s \pm} \\
& +\sum_{n=1}^{N_{s}^{m}} \bar{r}_{n}^{s \pm} \operatorname{grad} \Psi_{n}^{s \pm} ; \mathbf{H}_{v}^{N}=\frac{j}{k} \operatorname{rot} \mathbf{E}_{v}^{N}, v=0, i, s \pm
\end{aligned}
$$

Заметим, что внутри оболочки $D_{s}$ электромагнитное поле строится как сумма „уходящих“ и „приходящих“ волн:

$$
\mathbf{E}_{s}^{N}=\mathbf{E}_{s+}^{T N}+\mathbf{E}_{s-}^{T N}+\mathbf{E}_{s+}^{L N}+\mathbf{E}_{s-}^{L N}, \operatorname{div} \mathbf{E}_{s \pm}^{T N}=0, \operatorname{rot} \mathbf{E}_{s \pm}^{L N}=0 .
$$

Построенные поля (9) удовлетворяют системе уравнений Максвелла граничной задачи (4) и условиям сопряжения на бесконечной поверхности подложки $\Sigma$. А неизвестные амплитуды ДИ $\left\{p_{m n}^{v}, q_{m n}^{v}, r_{n}^{\nu} ; \bar{p}_{m n}^{s \pm}, \bar{r}_{n}^{s \pm}\right\}$ определяются из условий сопряжения на поверхностях $\partial D_{i, s}$.

Численный алгоритм строится по схеме, изложенной в [17]. Используя обобщенный метод коллокаций [25], фурье-гармоники полей (9) и внешнего возбуждения сшиваются на образующих поверхностей вращения, а контроль погрешности решения осуществляется вычислением невязки в промежуточных точках. Подобный подход позволяет обеспечить устойчивость вычислительной схемы при увеличении числа точек коллокаций и ДИ. Здесь следует подчеркнуть, что основная трудность при реализации схемы с учетом ОНО заключается в существенном различии значений продольного и поперечного волновых чисел. Это отношение $\left|k_{L} / k_{T}\right|$ может доходить до двух порядков в области резонансных длин волн. Таким образом, внутри плазмонного металла оказываются два поля с совершенно различным поведением. Однако эти сложности в рамках численной схемы МДИ компенсируются выбором различного числа ДИ для представления продольного и поперечного полей.

Для вычисления характеристик рассеяния в дальней зоне нам понадобится диаграмма направленности рассеянного поля $\mathbf{F}(\theta, \varphi)$, которая определяется в верхнем полупространстве как

$$
\begin{aligned}
\mathbf{E}_{0}(\mathbf{r}) /\left|\mathbf{E}^{0}(z=0)\right| & =\frac{\exp \left\{-j k_{0} r\right\}}{r} \mathbf{F}(\theta, \varphi)+O\left(1 / r^{2}\right), \\
r & \rightarrow \infty, z>0 .
\end{aligned}
$$


Тогда $(\theta, \varphi)$-компоненты диаграммы на единичной полусфере для Рполяризации принимают вид

$$
\begin{aligned}
F_{\theta}^{P}(\theta, \varphi) & =j k_{0} \sum_{m=0}^{M} \cos ((m+1) \varphi)(j \sin \theta)^{m} \\
& \times \sum_{n=1}^{N_{0}^{m}}\left\{p_{n m}^{0}\left[\bar{G}_{n}^{e} \cos \theta+j k_{0} \bar{g}_{n}^{e} \sin ^{2} \theta\right]+q_{n m}^{0} \bar{G}_{n}^{h}\right\} \\
& \left.-j \frac{k_{0}}{\varepsilon_{0}} \sin \theta \sum_{n=1}^{N_{0}^{0}} r_{n}^{0} \bar{G}_{n}^{h}\right\}, \\
F_{\varphi}^{P}(\theta, \varphi)= & -j k_{0} \sum_{m=0}^{M} \sin ((m=1) \varphi)(j \sin \theta)^{m} \sum_{n=0}^{N_{0}^{m}}\left\{p_{n m}^{0} \bar{G}_{n}^{e}\right. \\
& \left.+q_{n m}^{0}\left[\bar{G}_{n}^{h} \cos \theta+j k_{0} \bar{g}_{n}^{h} \sin ^{2} \theta\right]\right\},
\end{aligned}
$$

где соответствующие спектральные функции $\bar{G}_{n}^{e h}, \bar{g}_{n}^{h}$ могут быть представлены как

$$
\begin{aligned}
\bar{G}_{n}^{e, h} & =\exp \left\{j k_{0} z_{n} \cos \theta\right\}+A_{11}^{e, h}\left(k_{0} \sin \theta\right) \\
& \times \exp \left\{-j k_{0} \cos \theta\right\}, z_{n}>0 \\
\bar{g}_{n}^{e, h}(\theta) & =j k_{0} \cos \theta v_{31}^{e, h}\left(k_{0} \sin \theta, z=0, z_{n}\right) .
\end{aligned}
$$

Определив амплитуды ДИ для рассеянного поля, можно легко вычислить компоненты диаграммы направленности $(10),(11)$ на единичной полусфере $\Omega^{+}=\{0 \leq \theta \leq \pi / 2 ; 0 \leq \varphi \leq 2 \pi\}$, а также поле (9) в непосредственной близости от слоистой частицы.

\section{Численные результаты}

Будем рассматривать слоистую сферическую частицу с фиксированным диаметром ядра $D=15 \mathrm{~nm}$, состоящем из $\mathrm{SiO}_{2}$ с индексом рефракции $n_{i}=1.46$ и золотой оболочкой, толщину которой обозначим как $d$. Пусть частица располагается на стеклянной подложке ВК7 с индексом рефракции $n_{1}=1.52$ в воде с индексом $n_{0}=1.33$. В расчетах частотная дисперсия золота учитывается в соответствии с экспериментальными результатами [26].

Нас будут интересовать как интенсивность рассеяния в дальней зоне:

$$
\sigma_{\mathrm{sc}}\left(\theta_{0}, \lambda\right)=\int_{\Omega^{+}}\left|F_{\theta}^{P}\left(\theta_{0}, \theta, \varphi\right)\right|^{2}+\left|F_{\varphi}^{P}\left(\theta_{0}, \theta, \varphi\right)\right|^{2} d \omega,
$$

так и характеристики поля в непосредственной близости от внешней оболочки слоя $\partial D_{s}$. А именно коэффициент усиления интенсивности поля

$$
E\left(\theta_{0}, \lambda\right)=\int_{\partial D_{s}}\left|\mathbf{E}_{0}^{N}+\mathbf{E}_{0}^{0}\right|^{2} d \sigma / \int_{\partial D_{s}}\left|\mathbf{E}_{0}^{0}\right|^{2} d \sigma .
$$

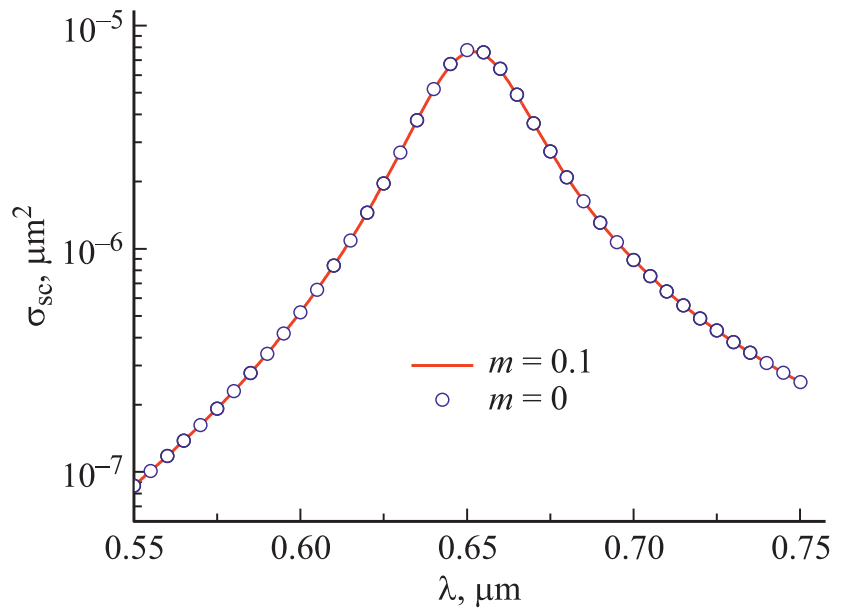

Рис. 1. Сечение рассеяния $\sigma_{\mathrm{sc}}(\lambda)$ по формуле (12) в зависимости от длины волны $\lambda$, толщина слоя $d=2 \mathrm{~nm}$, угол падения волны $\theta_{0}=45^{\circ}$. Сплошная кривая $m=0,1-$ учитываются диполи и квадруполи, кружки $m=0$ - только диполи.

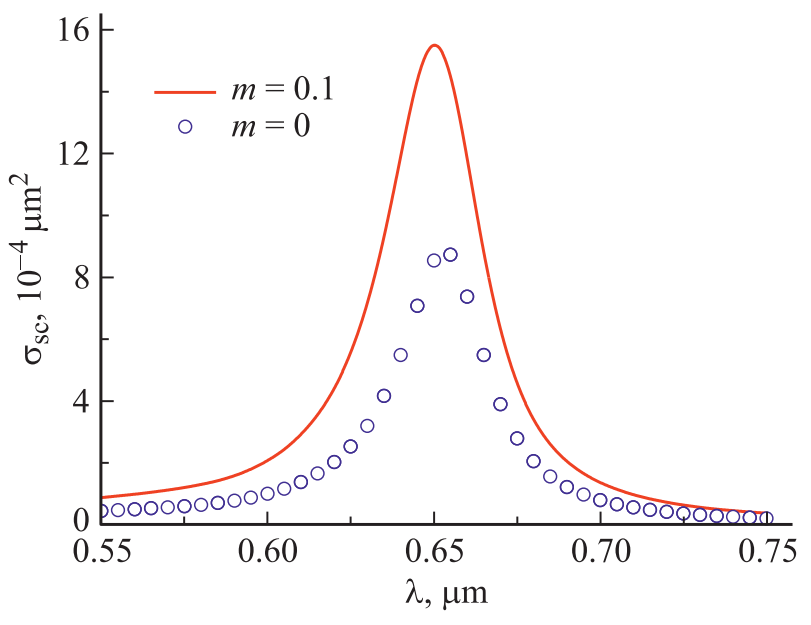

Рис. 2. То же, что и на рис. 1 , но для сечения поглощения $\sigma_{\mathrm{abs}}(\lambda)$ по формуле (14).

и сечение поглощения

$$
\sigma_{\mathrm{abs}}\left(\theta_{0}, \lambda\right)=-\operatorname{Re} \int_{\partial D_{s}}\left(\mathbf{E}_{0}^{N}+\mathbf{E}_{0}^{0}\right) \times\left(\mathbf{H}_{0}^{N}+\mathbf{H}_{0}^{0}\right)^{*} d \sigma .
$$

Размерности интенсивности и сечения поглощения равны $\mu \mathrm{m}^{2}$.

В наноплазмонике зачастую используют квазистационарное или дипольное приближения для анализа оптических свойств наночастиц [27]. Результаты, представленные на рис. 1,2, призваны подчеркнуть важность строгого учета взаимодействия частицы с подложкой, а также неприменимость использования дипольного приближения в этом случае. А именно подчеркнуть различие в точности аппроксимации ближнего и дальнего полей. Для этого выберем толщину слоя $d=2 \mathrm{~nm}$ и наклонный угол падения волны $\theta_{0}=45^{\circ}$. Все приведенные 
результаты соответствуют локальному случаю. В данном случае для аппроксимации поля плоской волны на $\partial D_{s} \mathrm{c}$ погрешностью $\approx 0.2 \%$ требуется учет фурье-гармоник с азимутальными числами $m=0,1$. При этом $m=0$ включает в себя вертикальные и горизонтальные диполи, а $m=0,1$ еще и горизонтальные квадруполи, распределенные вдоль оси вращения. На рис. 1 приведены результаты сравнения $\sigma_{\mathrm{sc}}(\lambda)$ для $m=0$ и $m=0,1$. Легко видеть, что эти результаты совпадают с графической точностью. На рис. 2 приведены результаты сравнения для $\sigma_{\mathrm{abs}}(\lambda)$, которые различаются почти в два раза. Это сравнение подчеркивает необходимость использования строгих подходов при анализе характеристик ближнего поля.

Перейдем к анализу влияния нелокальности на коэффициент усиления (13) и сечение поглощения (14). Для золота соответствующие квантовые параметры, необходимые для вычисления нелокальных величин $\varepsilon_{L}$ и $k_{L}$, выбраны равными [14]:

$$
\begin{gathered}
\hbar \omega_{p}=8.99 \mathrm{eV}, \hbar \gamma=0.025 \mathrm{eV} \\
\nu_{F}=1.39 \cdot 10^{12} \mu \mathrm{m} / \mathrm{s}, \quad D=9.62 \cdot 10^{8} \mu \mathrm{m}^{2} / \mathrm{s} .
\end{gathered}
$$

Задавая длину волны внешнего возбуждения $\lambda$, вычисляя соответствующее значение $\omega$, легко определить значения нелокальных параметров $\varepsilon_{L}$ и $k_{L}$.

На рис. 3, 4 приведены сравнительные результаты расчета коэффициента усиления и сечения поглощения для пленки толщиной $d=2$ и $3 \mathrm{~nm}$ в зависимости от угла падения плоской волны $\theta_{0}$ при фиксированных длинах волн. Видно, что обе величины монотонно возрастают при увеличении угла падения. В то же время учет ЭН приводит к существенному снижению величин максимумов. Например, для $d=2 \mathrm{~nm}$ у $\sigma_{\mathrm{abs}}$ оно составляет $33 \%$, a у $E-$ почти $50 \%$.

На рис. 5, 6 приведены те же величины, но в частотной области при фиксированном угле падения $\theta_{0}=45^{\circ}$. Как

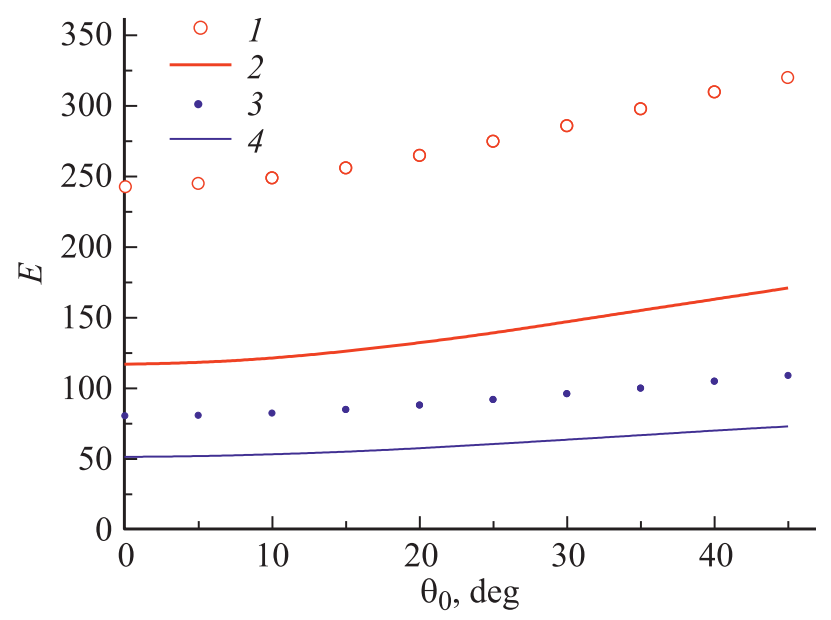

Рис. 3. Коэффициент усиления $E$ по формуле (13) в зависимости от угла падения волны $\theta_{0}: d=2 \mathrm{~nm}, \lambda=650 \mathrm{~nm}$, кружки 1 - локальный случай, сплошная кривая $2-$ с учетом ЭН; $d=3 \mathrm{~nm}, \lambda=600 \mathrm{~nm}$, точки 3 - локальный случай, сплошная кривая $4-$ с учетом ЭН.

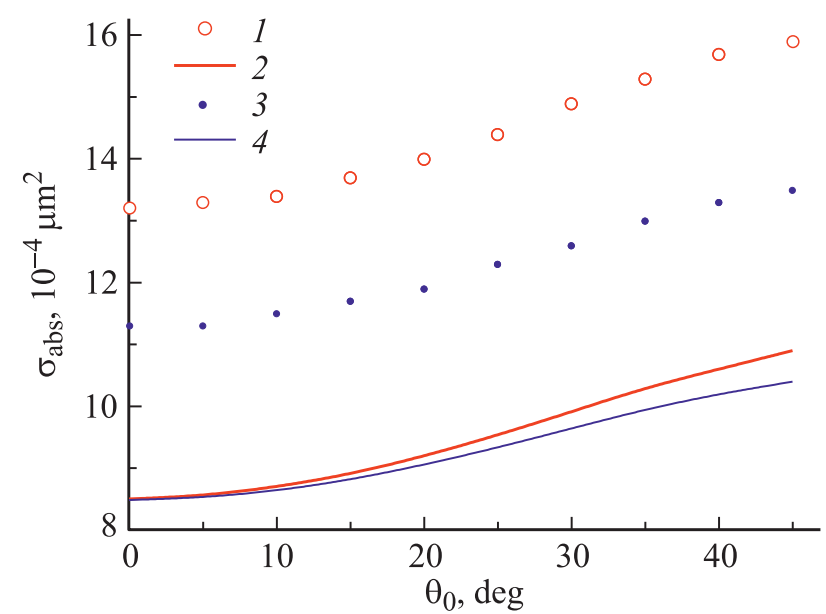

Рис. 4. То же, что и на рис. 3 , но для сечения поглощения $\sigma_{\mathrm{abs}}$ по формуле (14).

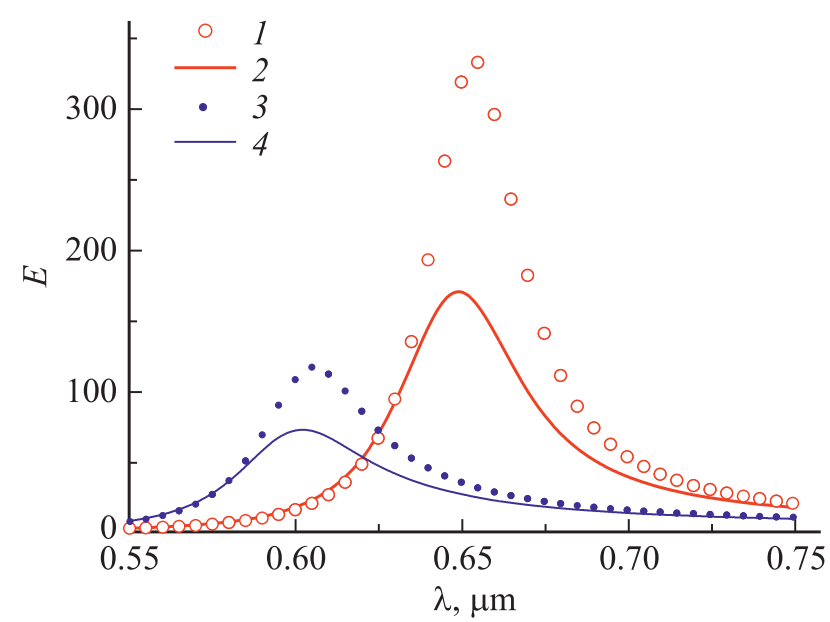

Рис. 5. Коэффициент усиления $E$ по формуле (13) в зависимости от длины волны $\lambda$, угол падения $\theta_{0}=45^{\circ}: d=2 \mathrm{~nm}$, кружки $1-$ локальный случай, сплошная кривая $2-$ с учетом ЭН; $d=3 \mathrm{~nm}$, точки 3 - локальный случай, сплошная кривая $4-$ с учетом ЭН.

и раньше, заметно, что учет ЭН приводит к снижению их значений. Причем сильнее это влияние проявляется для коэффициента усиления. На рисунках также заметен небольшой сдвиг плазмонного резонанса в область коротких длин волн.

Рис. 7,8 посвящены случаю возбуждения слоистой частицы неизлучающими волнами, когда плоская волн распространяется из призмы в верхнее полупространство, заполненное водой. Неизлучающие волны широко используются в современной оптике $[6,7,17]$. В данном случае область неизлучающих волн располагается перед углом, равным $\theta_{c}=118.7^{\circ}$. Мы выбрали угол падения волны $\theta_{0}=116.7^{\circ}$. В этом случае удается увеличить значения $E$ почти на $30 \%$, а сечение поглощения - более чем в 4 раза по сравнению со случаем падения волны сверху (рис. 5,6). При этом снова учет ЭН приводит к 


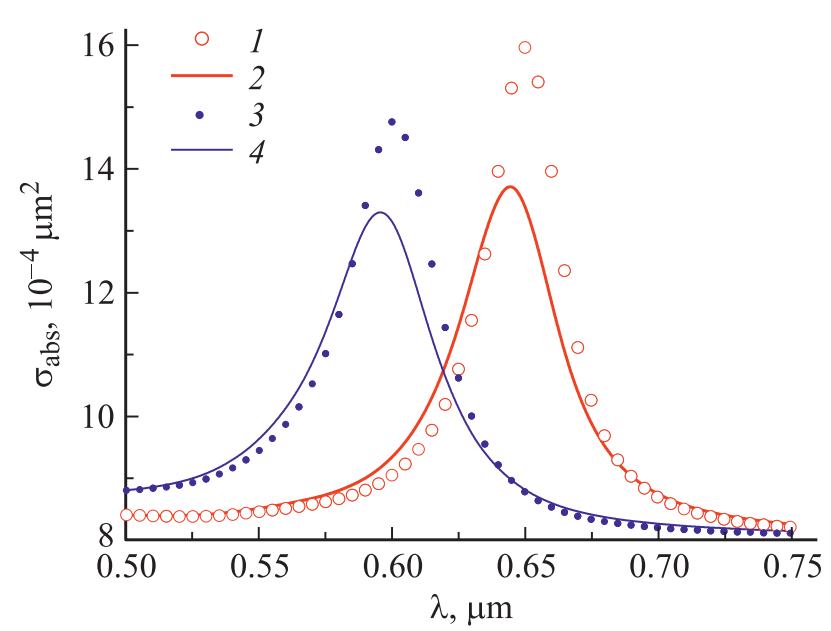

Рис. 6. То же, что и на рис. 5 , но для сечения поглощения $\sigma_{\mathrm{abs}}$ по формуле (14).

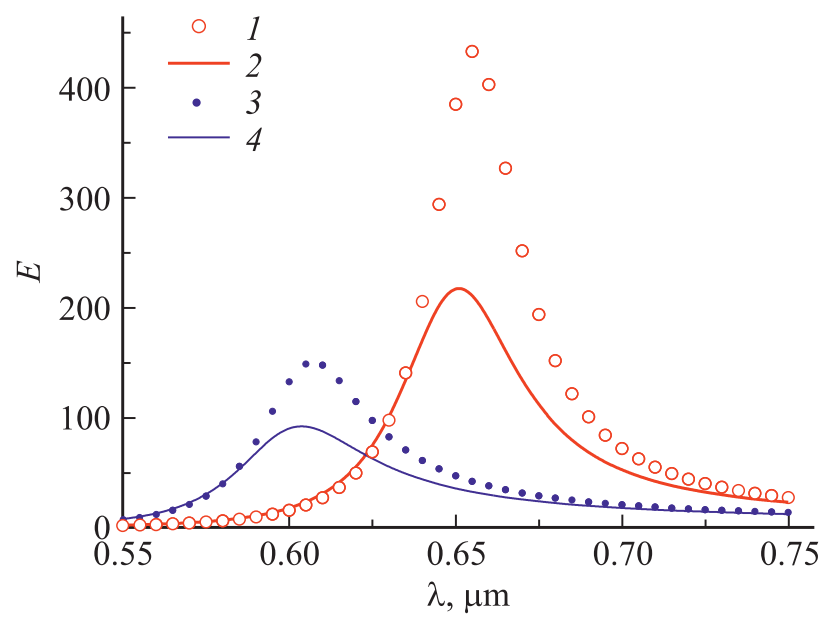

Рис. 7. Коэффициент усиления $E$ по формуле (13) в зависимости от длины волны $\lambda$, угол падения $\theta_{0}=116.7^{\circ}-$ область неизлучающих волн: $d=2 \mathrm{~nm}$, кружки $1-$ локальный случай, сплошная кривая $2-$ с учетом ЭН; $d=3 \mathrm{~nm}$, точки $3-$ локальный случай, сплошная кривая $4-$ с учетом ЭН.

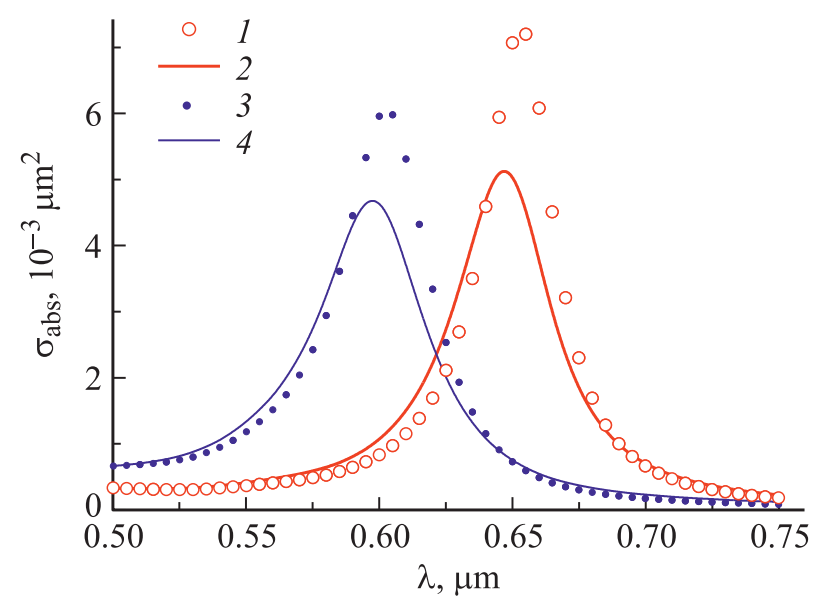

Рис. 8. То же, что на рис. 7, но для сечения поглощения $\sigma_{\mathrm{abs}}$ снижению максимумов величин и небольшому сдвигу в коротковолновую область.

\section{Финансирование работы}

Работа выполнена при финансовой поддержке Российского фонда фундаментальных исследований (проект № 20-01-00558).

\section{Список литературы}

[1] Xu D., Xiong X., Wu L. etal. // Adv. Opt. Photon. 2018. V. 10. N 4. P. 703. doi 10.1364/AOP.10.000703

[2] Zhang S., Geryak R., Geldmeier J., Kim S., Tsukruk V.V. // Chem. Rev. 2017. V. 117. P. 12942. doi 10.1021/acs.chemrev.7b00088

[3] Kalambate P.K., Dhanjai Huang Z., Li Y. etal. / Trends Analyt. Chem. 2019. V. 115. P.1 47-161. doi 10.1016/j.trac.2019.04.002

[4] Evlyukin A., Nerkararyan K.V., Bozhevolnyi S.I. // Opt. Express. 2019. V. 27. P. 17474. doi 10.1364/OE.27.017474

[5] Балыкин В.И. //УФН. 2018. Т. 188. № 9. С. 935. doi 10.3367/UFNr.2017.09.038206

[6] Sidorenko I., Nizamov Sh., Hergenr?de R., Zybin A. etal. // Microchim. Acta. 2016. V. 183. P. 101. doi 10.1007/s00604015-1599-0

[7] Avşar D., Ertürk H., Mengüç M.P. // Mater. Res. Express. 2019. V. 6. P. 065006. doi 10.1088/2053-1591/ab07fd

[8] Barbry M., Koval P., Marchesin F., Esteban R. etal. // Nano Lett. 2015. V. 15. P. 3410. doi 10.1021/acs.nanolett.5b00759

[9] David C., García de Abajo F.J. // J. Phys. Chem. C. 2011. V. 115. P. 19470. doi 10.1021/jp204261u

[10] Ciraci C., Pendry J.B., Smith D.R. // Chem. Phys. Chem. 2013. V. 14. P. 1109. doi 10.1002/cphc.201200992

[11] Derkachova A., Kolwas K., Demchenko I. // Plasmonics. 2016. V. 11. P. 941. doi 10.1007/s11468-015-0128-7

[12] Mortensen N.A., Raza S., Wubs M., Sфndergaard T., Bozhevolnyi S.I. // Nature Commun. 2014. V. 5. P. 3809. doi 10.1038/ncomms4809

[13] Tserkezi C., Stefanou N., Wubs M., Mortensen N.A. // Nanoscale. 2016. V. 8. P. 17532. doi 10.1039/C6NR06393D

[14] Wubs M., Mortensen A. // Quantum Plasmonics / Ed. by Bozhevolnyi S.I. et al. Springer, Switzerland. 2017. P. 279-302. doi 10.1007/978-3-319-45820-5_12

[15] Eremina E., Eremin Y., Wriedt T. // J. Mod. Opt. 2011. V. 58. P. 384. doi 10.1080/09500340.2010.515751

[16] Doicu A., Eremin Yu., Wriedt T. Acoustic and Electromagnetic Scattering Analysis Using Discrete Sources. Academic Press, San Diego, 2000.

[17] Гришина Н.В., Еремин Ю.А., Свешников А.Г. // Опт. и спектр. 2013. Т. 115. № 1. С. 133-139. doi 10.7868/S0030403413070076; Grishina N.V., Eremin Yu.A., Sveshnikov A.G. // Opt. Spectrosc. 2013. V. 115. № 1. C. 136. doi 10.1134/S0030400X13070072

[18] Еремин Ю.А., Свешников А.Г. // ЖВМиМФ. 2019. Т. 59. № 12. C. 2175. doi 10.1134/S0044466919100065; Eremin Yu.A., Sveshnikov A.G. // Comput. Math. Math. Phys. 2019. V. 59. N 12. P. 2164. doi 10.1134/S0965542519100063

[19] Eremin Yu., Doicu A., Wriedt T. // J. Quant. Spectr. Rad. Trans. 2019. V. 235. P. 300. doi 10.1016/j.jqsrt.2019.07.012 
[20] Лифиии Е.М., Питаевский Л.П. Физическая кинетика. М.: Наука, 1978. С. 167.

[21] Doicu A., Eremin Yu., Wriedt T. // J. Quant. Spectr. Rad. Trans. 2020. V. 242. P. 106756. doi $10.1016 /$ j.jqsrt.2019.106756

[22] Jerez-Hanckes C., Nédélec J.C. // Commun. Comput. Phys. 2012. V. 11. N 2. P. 629. doi 10.4208/cicp.231209.150910s

[23] Гришина Н.В., Еремин Ю.А., Свешников А.Г. // Опт. и спектр. 2012. Т. 113. № 4. С. 484; Grishina N.V., Eremin Yu.A., Sveshnikov A.G. // Opt. Spectrosc. 2012. V. 113. N 4. P. 440. doi 10.1134/S0030400X12100049

[24] Борн М., Воль $\oint$ Э. Основы оптики. М.: Наука, 1973. 713 с. Born M., Wolf E. Principles of Optics. N Y.: Pergamon Press, 1964.

[25] Бахвалов Н.С. Численные методы. М.: Наука, 1975. 632 с.

[26] Johnson P.B., Christy R.W. // Phys. Rev. B. 1972. V. 6. P. 4370.

[27] Khlebtsov N.G., Khlebtsov B.N. // J. Quant. Spectr. Rad. Trans. 2017. V. 190. P. 89. doi 10.1016/j.jqsrt.2017.01.027 